\title{
A formula for the normal subgroup growth of Baumslag-Solitar groups
}

\author{
J. O. Button \\ Selwyn College \\ University of Cambridge \\ Cambridge CB3 9DQ \\ U.K. \\ jb128@dpmms.cam.ac.uk
}

\begin{abstract}
We give an exact formula for the number of normal subgroups of each finite index in the Baumslag-Solitar group $B S(p, q)$ when $p$ and $q$ are coprime. Unlike the formula for all finite index subgroups, this one distinguishes different Baumslag-Solitar groups and is not multiplicative. This allows us to give an example of a finitely generated profinite group which is not virtually pronilpotent but whose zeta function has an Euler product.
\end{abstract}

\section{Introduction}

A finitely generated group $G$ has finitely many subgroups $a_{n}(G)$ of each finite index $n$ and hence finitely many normal subgroups $a_{n}^{\triangleleft}(G)$. If $R$ is the finite residual of $G$, that is the intersection of all finite index subgroups, then $a_{n}(G)=a_{n}(G / R)$ and $a_{n}^{\triangleleft}(G)=a_{n}^{\triangleleft}(G / R)$ by the correspondence theorem, so it is enough to consider only residually finite groups. There has been much recent activity in this area on the behaviour and asymptotic growth of these functions. There has also been work on obtaining exact formulae for $a_{n}(G)$ when $G$ lies in particular classes of groups. However much less is known about exact formulae for $a_{n}^{\triangleleft}(G)$. The only cases we can find in the literature 
are

(1) Some finite groups.

(2) Some abelian groups (where $a_{n}(G)=a_{n}^{\triangleleft}(G)$ anyway).

(3) Some torsion-free nilpotent groups of class 2 in [5]. Actually the normal zeta functions $\zeta_{G}^{\triangleleft}(s)$ are given from which we can deduce $a_{n}^{\triangleleft}(G)$.

(4) The 17 wallpaper groups have $a_{n}^{\triangleleft}(G)$ (or rather $\left.\zeta_{G}^{\triangleleft}(s)\right)$ listed in [2]. These groups are all finite extensions of the free abelian group of rank 2 .

(5) Groups $G$ where $G / R$ is in (1) to (4).

In this note we give a formula for the famous class of 2 generator 1 relator groups called the Baumslag-Solitar groups. These were introduced in [1] and the group $B S(p, q)$ has presentation

$$
\left\langle t, a \mid t a^{p} t^{-1}=a^{q}\right\rangle \text { for } p, q \in \mathbb{Z}-\{0\} .
$$

We consider the case when $p$ and $q$ are coprime and prove that

$$
a_{n}^{\triangleleft}(B S(p, q))=\sum_{\substack{d|n \\ d| q^{(n / d)}-p^{(n / d)}}} \operatorname{gcd}(d, q-p) .
$$

In [4] similar looking formulae in the case of all finite index subgroups were established for the same groups:

$$
a_{n}(B S(p, q))=\sum_{\substack{d \mid n \\ \operatorname{gcd}(d, p q)=1}} d .
$$

(In fact this formula in the case $p=1$ was earlier given in [10] Theorem 2.) Thus it is clear from (2) that $a_{n}(B S(p, q))$ is indistinguishable from $a_{n}\left(B S\left(p^{\prime}, q^{\prime}\right)\right)$ when $p q$ and $p^{\prime} q^{\prime}$ have the same prime factors but we show that the sequence $a_{n}^{\triangleleft}(B S(p, q))$ uniquely determines the Baumslag-Solitar group (and indeed $p$ and $q$ up to the obvious changes of swapping $p$ and $q$ and replacing them both with their negatives). We also prove that the sequence $a_{n}^{\triangleleft}(B S(p, q))$ is not multiplicative, unlike $a_{n}(B S(p, q))$. In [2] it is asked whether a profinite group whose zeta function has an Euler product is virtually pronilpotent. We show that this is not the case for the profinite completion of $B S(1, q)$ when $q \neq \pm 1$. Finally we work out which finitely generated groups $G$ have $a_{n}(G)=a_{n}^{\triangleleft}(G)$ for all $n$. 


\section{The formula}

Let $G$ be the Baumslag-Solitar group $B S(p, q)$ where $p$ and $q$ are coprime. The key point which allows us to enumerate the finite images of $G$, and hence the finite index normal subgroups, is the following:

Proposition 2.1 If $G=B S(p, q)$ for $p$ and $q$ coprime then the quotient of $G$ by its finite residual is metabelian.

Proof. This is probably known but an interesting way to see this is to recall that the proof of Malce'ev's result that a finitely generated residually finite group is Hopfian actually shows that any element in $\operatorname{ker}(\theta)$ for $\theta$ a surjective homomorphism of a finitely generated group $G$ is also in the finite residual $R$. Therefore on taking the famous homomorphism $\theta(t)=t, \theta(a)=a^{p}$ which is onto if $\operatorname{gcd}(p, q)=1$, we have that for any $j \geq 1$ the commutator $\left[t^{j} a t^{-j}, a\right]$ is in $\operatorname{ker}\left(\theta^{j}\right)$ and so the normal closure of $a$ in $G / R$, which is generated by the elements of the form $t^{k} a t^{-k}$ for $k \in \mathbb{Z}$, is abelian.

Of course if $p=1$ then $G$ is metabelian anyway, and residually finite by a result of Philip Hall but otherwise we have a non-ascending HNN extension of $\mathbb{Z}$ so $G$ will contain a non-abelian free group.

This gives us

Proposition 2.2 If $F$ is a finite image of $G=B S(p, q)$ for $p$ and $q$ coprime then $F$ is metacyclic with the order of a in $F$ being coprime to $p$ and to $q$.

Proof. Suppose we have $\theta: G \rightarrow F$. Then $H=\theta\left(\left\langle a^{p}\right\rangle\right)$ is conjugate in $F$ to $\theta\left(\left\langle a^{q}\right\rangle\right)$. If $H$ is strictly contained in $\theta(\langle a\rangle)$ then by [7] we have that $G$ is large. This happens if and only if $G / R$ is large but from above it is metabelian. (Another way of seeing that $G$ is not large is to use [3] Example 3.2 which shows that no finite index subgroup of $G$ has a homomorphism onto $\mathbb{Z} \times \mathbb{Z}$.)

Consequently we have $\theta\left(\left\langle a^{p}\right\rangle\right)=\theta\left(\left\langle a^{q}\right\rangle\right)=\theta(\langle a\rangle)=H$ and if the order of $x=\theta(a)$ is $d$ then $p$ and $q$ are both coprime to $d$. As $x^{p}$ and $x^{q}$ are generators of $H$ and $y x^{p} y^{-1}=x^{q}$ where $y=\theta(t)$, we see that the cyclic group $H$ is normal in $F$ and $F / H=\langle y H\rangle$ is also cyclic.

We can now deduce our main result. 
Theorem 2.3 If $p$ and $q$ are coprime then

$$
a_{n}^{\triangleleft}(B S(p, q))=\sum_{\substack{d|n \\ d| q^{(n / d)}-p^{(n / d)}}} g c d(d, q-p) .
$$

Proof. We count normal subgroups $N$ of index $n$ in $G=B S(p, q)$ where $x$ has exact order $d$ in the quotient. Fix $n$ and a factor $d$ and set $n=c d$. First let us consider the case where $p=1$. We have that the group with presentation

$$
\left\langle x, y \mid x^{d}, y x y^{-1}=x^{q}, y^{c}=x^{s}\right\rangle
$$

for some $s$ defined modulo $d$ surjects to $F=G / N$ as these relations all hold in $F$, where the last comes from the fact that $F / H$ is generated by $y H$ and has order $c$. As $y^{c} x y^{-c}=x$ we must require of $c$ that $d$ divides $q^{c}-1$ or else $x$ would not have order $d$. Also $y x^{s} y^{-1}=x^{s}$ implies that $d$ divides $s(q-1)$ for the same reason. Now with these conditions on $c$ and $s$ we have that the above presentation is a metacyclic group of order $c d$ by [6] Chapter 3 Theorem 1, thus this group is equal to $F$. Clearly $s$ is only defined modulo $d$ so the number of allowable choices for $s$ is the number of integers $s$ from 1 to $d$ such that $s(q-1)$ is 0 modulo $d$ which is $\operatorname{gcd}(d, q-1)$. Moreover different choices $s, s^{\prime}$ modulo $d$ give rise to different normal subgroups $N$ of $G$, or else we would have $y^{c}=x^{s}=x^{s^{\prime}}$ in $G / N$ which again contradicts the order of $x$.

Now take a general $p$ and again fix $n$ and $d$. From Proposition 2.2 we have that $d$ and $p$ are coprime so that in any finite image $F$ of order $n$ in which $x$ has order $d$ we can replace $x$ with $u=x^{p}$, which also generates the normal subgroup $H$ of $F$, to obtain the relation $y u y^{-1}=u^{r}$ where $q \equiv p r$ modulo $d$. We can now follow the argument just as before to obtain the claimed formula with $p$ replaced by 1 and $q$ by $r$. However $\operatorname{gcd}(d, q-p)=\operatorname{gcd}(d, r-1)$ and $d$ divides $q^{c}-p^{c}$ if and only if it divides $r^{c}-1$, so we are done.

\section{Consequences}

It is pointed out in [4] (and in [10] for $p=1$ ) that the formula (2) for all finite index subgroups only depends on the prime factors of $p q$ and so, as the abelianisation $G / G^{\prime}$ of a finitely generated group is the same as that for $G / R$, we can create examples of infinitely many finitely generated (and 
even residually finite) groups $B S(p, q)$ (respectively $B S(p, q) / R$ ) which are not isomorphic but which are isospectral, that is the sequences $a_{n}(G)$ are the same. However in the case of normal subgroups the sequence $a_{n}^{\triangleleft}(B S(p, q))$ determines $p$ and $q$.

Corollary 3.1 If $p$ and $q$ are coprime with $q \geq|p|$ then the sequence $a_{n}^{\triangleleft}(B S(p, q))$ uniquely determines $p$ and $q$.

Proof. If $a_{n}^{\triangleleft}(B S(p, q))=a_{n}^{\triangleleft}\left(B S\left(p^{\prime}, q^{\prime}\right)\right)$ with $q-p>q^{\prime}-p^{\prime} \geq 0$ then $a_{q-p}^{\triangleleft}(B S(p, q))=\sigma(q-p)$ for $\sigma$ the sum of divisors function. As we always have $a_{n}^{\triangleleft}(B S(p, q)) \leq \sigma(n)$ and strict inequality if $|q-p|<n$, we see that $a_{q-p}^{\triangleleft}\left(B S\left(p^{\prime}, q^{\prime}\right)\right)<\sigma(q-p)$. Consequently we assume $q-p=q^{\prime}-p^{\prime} \geq 1$ as $q=p$ implies $p, p^{\prime}, q, q^{\prime}$ are all 1 . Let us take an odd prime $l$ with $l^{m}$ dividing $q+p$ but not $q^{\prime}+p^{\prime}$. Then evaluating $a_{2 l^{m}}^{\triangleleft}(B S(p, q))$ involves summing over the odd divisors $l^{i}$ and the even divisors $2 l^{i}$ of $2 l^{m}$. In the odd case we obtain non-zero terms each time $l^{i}$ divides $q^{2 l^{m-i}}-p^{2 l^{m-i}}$, which is always because $q+p$ does too, and these terms are always 1 because $p$ and $q$ are coprime. In the even case we pick up contributions when $2 l^{i}$ divides $q^{l^{m-i}}-p^{l^{m-i}}$. However if $l$ divides this then it divides $q-p$ so the only non-zero term we have here comes from 2 if $q-p$ is even. As for $a_{2 l^{m}}^{\triangleleft}\left(B S\left(p^{\prime}, q^{\prime}\right)\right)$, first note that if we pick up a term $\operatorname{gcd}\left(d, q^{\prime}-p^{\prime}\right)$ then it has the same value as for $B S(p, q)$ so we just need to show that we end up with less non-zero terms. But we have the same contribution from the even divisors and we do not get $l^{m}$ dividing $q^{\prime 2}-{p^{\prime}}^{2}$ so this term of the sequence is strictly less.

This covers all cases except when $q+p=2^{s} u>q^{\prime}+p^{\prime}=2^{t} u$ for $u$ odd where we must have $t \geq 2$ and $q-p$ is 2 modulo 4 . We similarly consider the $2^{s+2}$-th term of each sequence and find that we pick up a term from $2^{s+1}$ in the first case but not in the second.

Note: It was shown in [9] that $B S(p, q)$ is isomorphic to $B S\left(p^{\prime}, q^{\prime}\right)$ if and only if $\left(p^{\prime}, q^{\prime}\right)=(p, q),(-p,-q),(q, p)$ or $(-q,-p)$. Whilst Corollary 3.1 could be regarded as providing an alternative proof (at least when $p$ and $q$ are coprime), surely the quickest way to see this is to note that the Alexander polynomial $\Delta(t)$ of $B S(p, q)$ is $p t-q$ and this is defined up to multiplication by $\pm t^{m}$ for $m \in \mathbb{Z}-\{0\}$. What Corollary 3.1 does establish is that for distinct Baumslag-Solitar groups $G$ with $p$ and $q$ coprime, we have that $G / R$ is distinct as well as the profinite completion.

Two finitely generated groups $G_{1}, G_{2}$ will be isospectral if their profinite completions are isomorphic, in which case we would have $a_{n}^{\triangleleft}\left(G_{1}\right)=a_{n}^{\triangleleft}\left(G_{2}\right)$ 
as well as the abelianisations $G_{1} / G_{1}^{\prime}$ and $G_{2} / G_{2}^{\prime}$ being equal because the finite abelian images have to be the same. The only isospectral groups known without isomorphic profinite completions seem to be the fundamental groups of the orientable and non-orientable surfaces of genus $g$, the example of $\mathbb{Z} \times$ $\mathbb{Z} \times C_{2}$ and $(\mathbb{Z} \times \mathbb{Z}) \rtimes C_{2}$ given in [2] after Theorem 1.4, and the BaumslagSolitar groups in [4]. However none of these examples provide isospectral pairs when we consider finite index normal subgroups, because of Corollary 3.1 for Baumslag-Solitar groups and by abelianising in the other cases. Thus we ask the following:

Question 3.2 Are there examples of finitely generated groups $G_{1}$ and $G_{2}$ with non-isomorphic profinite completions but with both $a_{n}\left(G_{1}\right)=a_{n}\left(G_{2}\right)$ and $a_{n}^{\triangleleft}\left(G_{1}\right)=a_{n}^{\triangleleft}\left(G_{2}\right)$ for all $n$ ?

Another property that we look for in the sequences $a_{n}(G)$ and $a_{n}^{\triangleleft}(G)$ is that they are multiplicative, namely $a_{m n}^{(\triangleleft)}=a_{m}^{(\triangleleft)} a_{n}^{(\triangleleft)}$ when $\operatorname{gcd}(m, n)=1$, as if so then we obtain an Euler product for the zeta function or normal zeta function of $G$. In [13] Theorem 1 it is shown that a finitely generated profinite group $G$ is pronilpotent if and only if $a_{n}^{\triangleleft}(G)$ is multiplicative. Consequently we can also answer the question after Proposition 1.5 in [2] which asks that if the zeta function of a profinite group $G$ has an Euler product then is $G$ virtually pronilpotent.

Theorem 3.3 The profinite completion of $B S(1, q)$ for $q \neq \pm 1$ has the property that its zeta function possesses an Euler product but it is not virtually pronilpotent.

Proof. We first show that for $p, q$ coprime we have $a_{n}(B S(p, q))$ is multiplicative but $a_{n}^{\triangleleft}(B S(p, q))$ is not (excepting of course $\left.B S(1,1)=\mathbb{Z} \times \mathbb{Z}\right)$. On taking the formula (2) for $a_{n}(B S(p, q))$, and ignoring the $\operatorname{gcd}(d, p q)=1$ condition in the sum, we have multiplicity of the sum of divisors function $\sigma(n)$ which is proved by multiplying the sums $\sigma(m)$ and $\sigma(n)$ together to obtain a new sum whose terms are exactly the divisors of $m n$. Now put the condition back in and mark the terms in $\sigma(m)$ and in $\sigma(n)$ that fail to be coprime to $p q$. When we multiply out to get $\sigma(m n)$, the terms in this sum with one or both factors marked are exactly the ones which are not coprime to $p q$. Hence by erasing the marked terms on both sides we have multiplicity and thus we obtain an Euler product for the zeta function of $B S(p, q)$ whenever $p$ and $q$ are coprime, and hence for its profinite completion. 
As for $a_{n}^{\triangleleft}(B S(p, q))$, let us take an odd prime $l$ dividing $p^{2}+q^{2}$, which will exist except when $(p, q)=(1, \pm 1)$ because $p^{2}+q^{2}$ is not 0 modulo 4 . Now $a_{l}^{\triangleleft}(B S(p, q))=1$ because $p^{2}+q^{2}$ and $q-p$ are coprime (apart from a possible factor of 2 ) and $a_{4}^{\triangleleft}(B S(p, q))$ is 1 if $q-p$ is odd, 3 if $q-p$ is 2 modulo 4 and 7 if $q-p$ is 0 modulo 4 . But as $l$ divides $q^{4}-p^{4}$ we have $a_{4 l}^{\triangleleft}(B S(p, q))$ is at least one more than these numbers. (For $B S(1,-1)$ we have $a_{2}^{\triangleleft}=3, a_{3}^{\triangleleft}=1$ and $a_{6}^{\triangleleft}=4$.)

Thus by Puchta's result in [13] we have that the profinite completion of $B S(p, q)$ is not pronilpotent unless $p=q=1$. Moreover on taking $p=1$ we have by [10] that the finite index subgroups of $B S(1, q)$ are isomorphic to $B S\left(1, q^{n}\right)$ for $n \geq 1$, so if $q \neq \pm 1$ then the profinite completion of $B S\left(1, q^{n}\right)$ is not virtually pronilpotent.

Alternatively we can show that the profinite completion of $B S(p, q)$ is not pronilpotent by directly finding a finite image which is not nilpotent. Although if $p \neq 1$ we cannot then use the finite index subgroup trick as above, an adaptation shows that the profinite completion is not virtually pronilpotent in all cases other than $B S(1, \pm 1)$, although if $p \neq 1$ we do not have an embedding of $B S(p, q)$ into its profinite completion. We outline the proof: if $H$ has finite index $k_{0}$ in $G=B S(p, q)$ for $q>|p|$ and all the finite images of $H$ are nilpotent then any finite image of $G$ with order more than $k_{0}$ must have a non-trivial element whose centraliser in $G$ has index at most $k_{0}$. But if we pick a prime $l>\max \left(q^{k_{0}}-p^{k_{0}}, k_{0}, q\right)$ and let $k>0$ be the first value where $l$ divides $q^{k}-p^{k}$ then $k>k_{0}$ and $G$ has the finite image

$$
F=\left\langle x, y \mid y x^{p} y^{-1}=x^{q}, x^{l}, y^{k}\right\rangle=C_{l} \rtimes C_{k} .
$$

But it is easily checked that $l$ being prime means that no non-trivial power of $x$ commutes with no non-trivial power of $y$ and this in turn implies that the centraliser of $x$ has index $k$ and all other centralisers have index $l$.

Although we have assumed throughout, as in [4], that $p$ and $q$ are coprime, it is reasonable to ask about the case of common factors. However any results will be vastly different because now $B S(p, q)$ is large by [3] Theorem 6 , as if $g=\operatorname{gcd}(p, q)$ then we have a surjection to the virtually free group $\mathbb{Z} * C_{g}$ on adding the relator $a^{g}$. This implies that it has subgroup growth of strict type $n^{n}$ and, by [11] Theorem 1, normal subgroup growth of strict type $n^{\log n}$. Both of these are the fastest possible types for a finitely generated group. Moreover 
although $B S(p, q)$ may still not be residually finite if common factors are present (as this happens if and only if $|p|=|q|$ ), we have by [8] Theorem 3.1 that every finite group is an upper section. Hence a large group $L$ (and any finite index subgroup of $L$ as this is also large) always has a finite non-soluble image and so the profinite completion of a large group is never prosoluble. Thus $a_{n}^{\triangleleft}(B S(p, q))$ is not multiplicative in this case by [13] and we can also show here that neither is $a_{n}(B S(p, q))$.

Proposition 3.4 If $G$ is a finitely generated group with $\lambda, \mu>0$ such that $a_{n}(G) \geq \lambda e^{\mu n}$ for all large $n$ then $a_{n}(G)$ cannot be multiplicative.

Proof. If so then we would have $a_{n(n+1)} \geq \lambda e^{\mu n(n+1)}$ but as $a_{n} \leq k n^{n d}$ if $G$ is a $d$-generator group (as this is also true for the free group of rank $d$ ), we obtain

$$
a_{n(n+1)} / a_{n(n+1)} \geq \lambda e^{\mu n(n+1)} / k^{2} n^{n d}(n+1)^{(n+1) d}
$$

which tends to infinity as $n$ does.

Corollary 3.5 If $p$ and $q$ are not coprime then $a_{n}(B S(p, q))$ is not multiplicative.

Proof. We have $a_{n}(B S(p, q)) \geq a_{n}\left(\mathbb{Z} * C_{g}\right)$ and without loss of generality we may assume that $g$ is a prime, in which case we have the lower bound $\lambda n e^{(1-1 / g) n(\log n-1)+n^{1 / g}}$ in [12].

We finish by giving the finitely generated groups such that $a_{n}(G)=$ $a_{n}^{\triangleleft}(G)$. Here we use the fact due to Dedekind and Baer that a non-abelian finite group $F$ has all its subgroups normal if and only if $F=Q \times A \times B$ where $Q$ is the quaternion group of order $8, A$ is an elementary abelian 2-group and $B$ is an abelian group of odd order.

Proposition 3.6 If $G$ is a finitely generated group where every finite index subgroup is normal then either $G / R$ is abelian and equal to $G / G^{\prime}$ or $G / R$ is finite and equal to $Q \times A \times B$ as above.

Proof. We have $R \leq G^{\prime}$ so let us assume throughout that $R<G^{\prime}$. Then $G$ has a finite index subgroup $N$ with $G / N$ non-abelian. Now if $G / G^{\prime}$ is infinite 
then we have $M$ with $G / M=C_{4}$ and so $G /(M \cap N)$ is a finite non-abelian group which surjects onto $C_{4}$, but as $Q / Q^{\prime}=C_{2} \times C_{2}$ we have $G /(M \cap N)$ has a subgroup which is not normal and so $G$ has such a finite index subgroup. But if $G / G^{\prime}$ is finite then there is a bound for the order of $F / F^{\prime}$ as $F$ ranges over the finite images of $G$ because $F$ must have the form above, and so there is a bound for the order of $F$. Thus $R$ has finite index in $G$ with $G / R$ equal to some $F$.

\section{References}

[1] G. Baumslag and D. Solitar, Some two-generator one-relator nonHopfian groups, Bull. Amer. Math. Soc. 68 (1962), 199-201.

[2] M.P.F.du Sautoy, J. J. McDermott and G.C.Smith, Zeta functions of crystallographic groups and analytic continuation, Proc. London Math. Soc. 79 (1999), 511-534.

[3] M. Edjvet and S. J. Pride, The concept of "largeness" in group theory II, in Groups - Korea 1983, Lecture Notes in Math. 1098, Springer, Berlin, 1984, pp. 29-54.

[4] E. Gelman, Subgroup growth of Baumslag-Solitar groups, J. Group Theory 8 (2005), 801-806.

[5] F. J. Grunewald, D. Segal and G. C. Smith, Subgroups of finite index in nilpotent groups, Invent. Math. 93 (1988), 185-223.

[6] D. L. Johnson, Topics in the theory of group presentations, London Math. Soc. Lecture Note Series 42, Cambridge University Press, Cambridge-New York, 1980.

[7] A. Lubotzky, Free Quotients and the first Betti number of some hyperbolic manifolds, Transform. Groups 1 (1996) 71-82.

[8] A. Lubotzky and D. Segal, Subgroup growth. Progress in Mathematics 212, Birkhaüser Verlag, Basel, 2003. 
[9] D. I. Moldavanskii, On the isomorphisms of the Baumslag-Solitar groups, Ukrain. Mat. Zh. 43 (1991), 1684-1686.

[10] D. I. Moldavanskii and N. Sibyakova, On the finite images of some onerelator groups, Proc. Am. Math. Soc. 123 (1995), 2017-2020.

[11] T.W. Müller and J.-C.Schlage-Puchta, Normal Subgroup of large groups, II, Arch. Math. 84 (2005), 289-291.

[12] M. Newman, Asymptotic formulas related to free products of cyclic groups, Math. Comp. 30 (1976), 838-846.

[13] J.-C. Puchta, Groups with multiplicative subgroup growth, Israel J. Math. 122 (2001), 149-156. 